\title{
Plasma Ion Implantation of Nitrogen into Silicon: High Resolution X-ray Diffraction
}

\author{
A. F. Beloto ${ }^{1}$, E. Abramof ${ }^{1}$, \\ M. Ueda ${ }^{2}$, L. A. Berni ${ }^{2}$, and G. F. Gomes ${ }^{2,3}$ \\ 1: Laboratório Associado de Sensores e Materiais, \\ Instituto Nacional de Pesquisas Espaciais, \\ C.P. 515 -12201-970 São José dos Campos, SP, Brazil \\ 2: Laboratório Associado de Plasma, \\ Instituto Nacional de Pesquisas Espaciais, \\ C.P. 515 -12201-970 São José dos Campos, SP, Brazil \\ 3: FAENQUIL, Lorena, SP, Brasil \\ Received February 8, 1999
}

\begin{abstract}
In the present study we use x-ray diffraction methods to characterize the surface of $S i$ wafers irradiated with nitrogen by Plasma Immersion Ion Implantation. This is a non-line-of-sight ion implantation method, which allows three-dimensional treatment of materials including semiconductors, metals and dielectrics. The atomic concentration profiles in the implanted Si wafers were measured by Auger electron spectroscopy. The (004) Si rocking curve ( $\omega$-scan) was measured in a high resolution x-ray diffractometer equipped with a $G e(220)$ four-crystal monochromator before and after implantation. A distortion of the $S i$ (004)-rocking curve was clearly observed for the as-implanted sample. This rocking curve was successfully simulated by dynamical theory of x-ray diffraction, assuming a Gaussian strain profile through the implanted region. The analysis made by x-ray diffraction and Auger electron spectroscopy revealed successful implantation of ions with accumulated nitrogen dose of $1.5 \times 10^{17} \mathrm{~cm}^{-3}$. The $S i$ wafers can be used as high sensitivity monitors in the Plasma Immersion Ion Implantation process, especially at the low dose range.
\end{abstract}

\section{Introduction}

Plasma Immersion Ion Implantation (PIII) is a novel ion implantation technique developed recently for the improvement of the surface properties of materials including semiconductors, metals and dielectrics [1]. It is inherently a non-line-of-sight ion implantation method which allows three-dimensional surface treatment of manufactured workpieces of large dimension and/or complex shapes, at high speed, in batch processing mode and in a cost-effective manner [2]. In PIII, the ions of interest are extracted directly from the plasma in which the samples to be processed are immersed, without the need of acceleration grids.

In this work, $S i$ wafers were implanted with nitrogen using the PIII technique. The wafers were characterized by Auger Electron Spectroscopy (AES) in order to determine the concentration profiles. The samples were analyzed by high-resolution x-ray diffraction before and after implantation. The profiles of the Si (004) rocking curves were simulated by dynamical theory of x-ray diffraction and compared with the measured data.

\section{Plasma Immersion Ion Im- plantation System}

Fig. 1 shows a diagram of the PIII system used. The plasma is produced by a DC glow discharge source located inside a vacuum chamber $\left(<10^{-3}\right.$ mbar $)$. The three-dimensional ion implantation is achieved by applying repetitively a negative high voltage pulse ( 7 to 15 $\mathrm{kV}, 5$ to $10 \mu$ s duration times, 5 to $20 \mathrm{~Hz}$ repetition frequencies) to the sheath formed between the plasma and the sample holder. While the voltage pulse is present, the sheath boundary propagates outward and before it reaches the chamber walls the high voltage is turnedoff, terminating the implantation process during that pulse [3]. The electron shower controls the plasma potential and the Langmuir probe is used to measure the plasma density. 


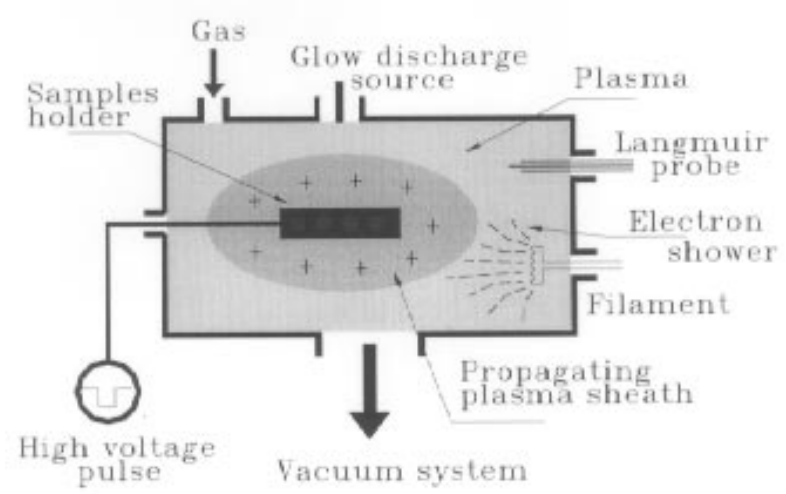

Figure 1. Schematic drawing of the DC glow discharge plasma ion immersion implantation system.

This system delivers plasmas with medium electron densities $\left(n_{e}>10^{10} \mathrm{~cm}^{-3}\right)$ low electron temperatures $\left(T_{e}<10 \mathrm{eV}\right)$, controlled plasma potentials $\left(\phi_{p}\right)$ between 0 and $350 \mathrm{~V}$, and with high stability for long operation times $(>50 \mathrm{~h})$. The ion temperature is much smaller than Te in such plasma. These characteristics allied with the possibility of low-pressure operation $\left(<10^{-3}\right.$ mbar $)$ are highly favorable for the PIII processing of materials.

\section{Nitrogen Implatation in Sil- icon}

We used $0.5-\mathrm{mm}$ thick, $1.5 \times 1.5 \mathrm{~cm}$ pieces of $\mathrm{p}$ type (100) Si wafer polished in one side and chemically cleaned just before their insertion into the vacuum chamber. They were fixed and masked adequately in a metallic sample holder.

The $S i$ wafers were implanted with the plasma potential controlled at $\phi_{p}=70 \mathrm{~V}$, with plasma density of $1.5 \times 10^{10} \mathrm{~cm}^{-2}$. The high voltage pulser was operated with peak voltage of $10 \mathrm{kV}, 6 \mu$ s pulse duration and repetition frequency of $20 \mathrm{~Hz}$. Based on the sheath propagation model, an estimated dose of $1.0 \times 10^{17} \mathrm{~cm}^{-2}$ is expected for $1500 \mathrm{~min}$. of implantation

\section{Auger Experiments}

Measurements of the concentration profiles of PIII nitrogen implanted samples were performed by Auger Electron Spectroscopy. The AES result is depicted in Fig. 2 for a $S i$ wafer implanted during $1500 \mathrm{~min}$.. It demonstrates the large concentration of nitrogen $(60 \%$ a.c.) implanted into the $S i$ lattice. The implanted nitrogen atoms reach depths down to $35 \mathrm{~nm}$, which correspond roughly to the range of $10 \mathrm{keV}, \mathrm{N}^{+}$ions in $S i$. From this AES measurement, it is possible to conclude that a retained nitrogen dose of about $1.5 \times 10^{17} \mathrm{~cm}^{-2}$ was reached.

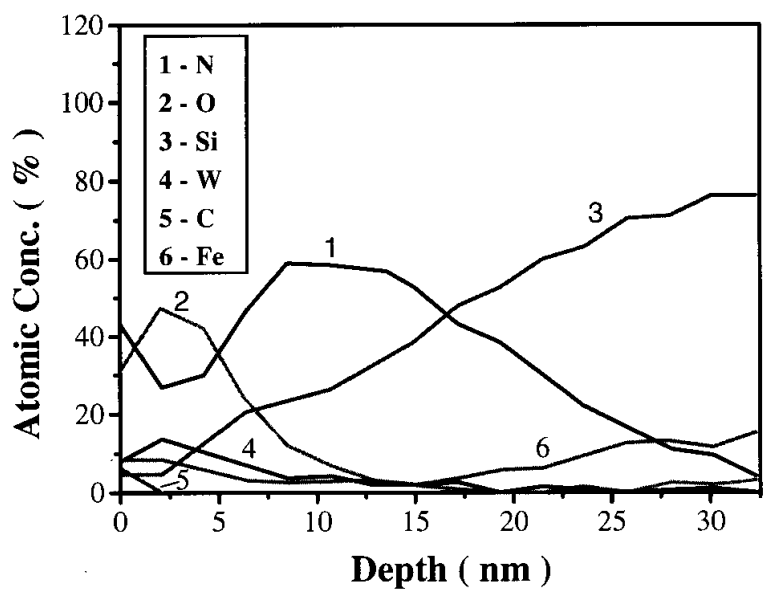

Figure 2. Concentration profiles of the nitrogen implanted $S i$ wafer (curve 1) measured with AES technique. Concentration profiles of impurities present in the implanted sample are also shown.

The oxygen peak concentration of $50 \%$ observed in the AES spectrum in the first $10 \mathrm{~nm}$ may have been formed by recoil implantation of oxygen from the $\mathrm{SiO}_{2}$ native film or from implantation of oxygen present as impurity in the discharge. This oxygen barrier is known to be crucial for a good retention of the implanted nitrogen [4]. The tungsten peak (10\% a.c.) observed near the surface comes from the heated tungsten filament.

\section{High Resolution x-Ray Diffraction Analysis}

The x-ray diffraction measurements were carried out in a Philips X'Pert diffractometer using $C u K \alpha_{1}$ radiation. The incident optics is equipped with a four-crystal $G e(220)$ monochromator which gives a horizontal divergence of 12 arcsec and a wavelength dispersion of less than $3 \times 10^{-5}$. An open detector is used in the secondary optics. $\omega$-Scans around the Si (004) diffraction peak with the detector fixed at the $2 \Theta$ Bragg angle (rocking curve) were performed on the $S i$ wafers before and after each implantation.

Fig. 3 shows the (004) diffraction peak of a $S i$ wafer before and after 1500 min. of nitrogen implantation. An evident distortion of the rocking curve caused by the implanted nitrogen atoms can be observed in comparison to the symmetrical rocking curve obtained for the same sample before implantation. Similar variations of x-ray (113) rocking curves in $S i$ wafers implanted with boron by plasma source ion implantation were reported previously [5]. 


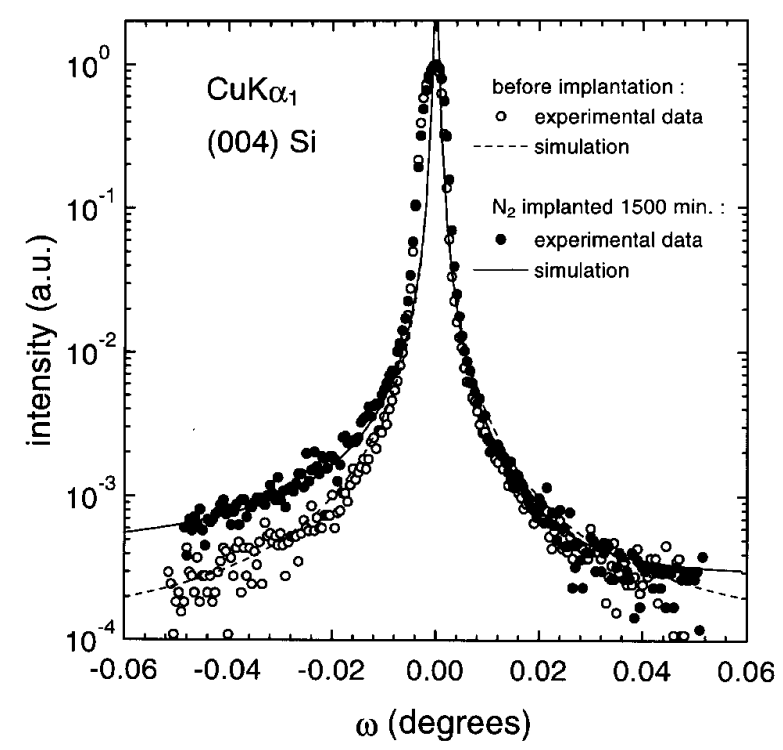

Figure 3. $\omega$-Scans around (004) Si diffraction peak before and after $1500 \mathrm{~min}$. of nitrogen implantation. The solid and dashed lines are simulations by dynamical theory of x-ray diffraction.

The (004) rocking curves were simulated by dynamical theory of x-ray diffraction based on Takagi-Taupin equations $[6,7]$. The implantation of ions in crystalline lattices creates strain in the implanted region. In our xray diffraction simulation, the strain profile through the implanted region is supposed to be given by a Gaussian distribution. The central peak position $(110 \mathrm{~nm})$ and the width $(140 \mathrm{~nm})$ of the Gaussian strain distribution used in the simulation of the implanted diffraction peak (solid line in Fig.3) were taken from the profile of nitrogen atoms obtained from the AES spectrum shown in Fig. 2. The maximum of the strain distribution was $2 \times 10^{-3}$. No strain is assumed in the rocking curve simulation of unimplanted $S i$ (dashed line in Fig. .3).

Results from the x-ray diffraction simulation showed that the rocking curves are much more sensitive to the depth of the implanted region than to the absolute value of the strain distribution An excellent agreement between the simulated and measured (004) rocking curve was obtained for the implanted sample. This agreement demonstrates that the AES measurements correlate very well with the $\mathrm{x}$-ray diffraction data.

\section{Conclusion}

$S i$ wafers were implanted successfully with nitrogen in a Plasma Ion Immersion Implantation system. They can be used as retained dose monitors in the range from $10^{15}$ to $10^{17} \mathrm{~cm}^{-2}$, for low voltage PIII implantation $(10 \mathrm{kV})$, extending the results of a previous work carried out for higher voltage PIII (50 to $100 \mathrm{kV}$ ) [8].

The AES measurements showed that the implanted nitrogen region extended to a depth of $35 \mathrm{~nm}$, which correspond roughly to the range of $10 \mathrm{keV}, N^{+}$ions in Si.

The rocking curve of the $S i$ (004) diffraction peak measured in a high-resolution x-ray diffraction system showed an evident distortion after the nitrogen PIII process in relation to the same sample without implantation. The rocking curve of the implanted sample was simulated with success assuming a Gaussian strain distribution in the implanted region taken from the AES nitrogen profile. The non-destructive $\mathrm{x}$-ray diffraction analysis performed in the implanted $S i$ wafers give useful information about the PIII process.

We gratefully thank Dr.R.Guenzel and Mrs. E.Quaritsch from Research Center Rossendorf, Dresden, Germany, who made the AES measurement of our samples possible. G.F.Gomes is supported by CAPES fellowship.

\section{References}

1. J.R. Conrad, J.L. Radtke, R.A. Dodd, F.J. Worzala and N.C. Tran, J. Appl. Phys., 62, 4591 (1987).

2. D.J. Rej, in Handbook of Thin Film Process Technology (IOP Publishing Ltd, 1996), pp. E2.3:1 $\mathrm{E} 2.3: 25$.

3. J.V. Mantese, I.G. Brown, N.C. Cheung and G.A. Collins, MRS Bulletin, 21, 52 (1996).

4. S. Parascandola and O. Kruse, Annual Report 1997, Institute of Ion Beam Physics and Materials Research, Dresden, Germany, pp 40-43.

5. D.L. Chapek, J.R. Conrad, R.J. Matyl, S.B. Felch, J. Vac. Sci. Technol., B 12, 951 (1994).

6. W.J. Bartels, J. Honstra, D.J.W. Lobeek, Acta Crystallogr. Sec., A 42, 539 (1986).

7. A. Pesek, P. Kastler, K. Lischka, L. Palmetshofer, Nucl. Instrum. Meth. B 80, 569 (1993).

8. J. Vajo, J.D. Williams, R. Wei, R. Wilson and J.M. Matossian, J. Appl. Phys., 76, 5666 (1994). 